\title{
Generalized results of majorization inequality via Lidstone's polynomial and newly Green functions
}

\author{
Nouman Siddique ${ }^{\mathrm{a}}$, Naveed Latif ${ }^{\mathrm{b}, *}$, Josip Pečarićc,d \\ ${ }^{a}$ Department of Mathematics, Govt. College University, Faisalabad 38000, Pakistan. \\ ${ }^{b}$ General Studies Department, Jubail Industrial College, Jubail Industrial City 31961, Kingdom of Saudi Arabia. \\ ${ }^{c}$ Faculty of Textile Technology Zagreb, University of Zagreb, Prilaz Baruna Filipovića 28A, 10000 Zagreb, Croatia. \\ ${ }^{d}$ RUDN University, 6 Miklukho-Maklay St, Moscow, 117198, Russia.
}

Communicated by M. Bohner

\begin{abstract}
Generalized results of majorization inequality are obtained by using newly Green functions defined in [N. Mahmood, R. P. Agarwal, S. I. Butt, J. Pečarić, J. Inequal. Appl., 2017 (2017), 17 pages] and Lidstone's polynomial. We find new upper bounds of Grüss and Ostrowski type. We give further results of majorization inequality by making linear functionals constructed on convex functions $\frac{f(x)}{x}$. Some applications are given.

Keywords: Classical majorization theorem, Fuchs's thorem, Lidstone's interpolating polynomial, Green Function for 'two point right focal' problem, Čebyšev functional, Grüss type upper bounds, Ostrowski-type bounds, convex function $f(x) / x$, n-exponentially convex function, mean value theorems, Stolarsky type means.
\end{abstract}

2010 MSC: 26D07, 26D15, 26D20, 26D99.

(C)2018 All rights reserved.

\section{Introduction}

The study of convex sets and convex functions is a comparatively recent development (see [6]). Although convexity appears implicitly much earlier (going back to work of Archimedes, in fact), the first papers on convex sets appeared at the end of nineteenth century. The characterizations of solutions of optimization problems, first appeared around the middle of the twentieth century. Starting in the 1970s, there has been considerable work on extending these methods to nonconvex functions.

Majorization: [17, p.319] $\mathbf{x}$ is said to majorize $\mathbf{y}$ (or $\mathbf{y}$ is said to be majorized by $\mathbf{x}$ ), in symbol, $\mathbf{x} \succ \mathbf{y}$, if

$$
\sum_{i=1}^{l} y_{[i]} \leqslant \sum_{i=1}^{l} x_{[i]}
$$

\footnotetext{
*Corresponding author

Email addresses: nouman6522@gmail.com (Nouman Siddique), naveed707@gmail.com (Naveed Latif), pecaric@element.hr (Josip Pečarić)
}

doi: $10.22436 /$ jnsa.011.06.08

Received: 2017-02-15 Revised: 2018-01-02 Accepted: 2018-03-08 
holds for $l=1,2, \ldots, m-1$ and

$$
\sum_{i=1}^{m} x_{i}=\sum_{i=1}^{m} y_{i}
$$

where $x_{[i]}$ and $y_{[i]}$ denote their decreasing order.

Because $x_{i}$ 's and $y_{i}$ 's are reordered decreasingly in the definition of majorization, their original order plays no role. Thus, the fact that these numbers are viewed as components of vectors is not important to the concept of majorization. But it is convenient to regards $\mathbf{x}=\left(x_{1}, x_{2}, \ldots, x_{n}\right)$ and $\mathbf{y}=\left(y_{1}, y_{2}, \ldots, y_{n}\right)$ are vectors so that the relationship $\mathbf{x}=\mathbf{y P}$ can be written with standard notation.

The following notion of Schur-convexity generalizes the definition of convex function via the notion of majorization (see [1]). The superb reference on the subject majorization is the monograph [16].

A function $F: S \subseteq \mathbb{R}^{n} \rightarrow \mathbb{R}$ is called Schur-convex on $S$ if

$$
\mathrm{F}(\mathbf{y}) \leqslant \mathrm{F}(\mathbf{x})
$$

for every $\mathbf{x}, \mathbf{y} \in \mathrm{S}$ such that

$$
\mathbf{y} \prec \mathbf{x} .
$$

A relation between one-dimensional convex function and m-dimensional Schur-convex function is included in the following Majorization theorem proved by G. H. Hardy, J. E. Littlewood, G. Pólya (see [8], [17, p. 333]).

Theorem 1.1 (Majorization Theorem). Let $\mathrm{I} \subset \mathbb{R}$ be an interval and $\mathbf{x}=\left(\mathrm{x}_{1}, \ldots, \mathrm{x}_{\mathrm{m}}\right), \mathbf{y}=\left(\mathrm{y}_{1}, \ldots, \mathrm{y}_{\mathrm{m}}\right) \in \mathrm{I}^{\mathrm{m}}$. Let $\mathrm{f}: \mathrm{I} \rightarrow \mathbb{R}$ be continuous function. Then a function $\mathrm{F}: \mathrm{I}^{\mathrm{m}} \rightarrow \mathbb{R}$, defined by

$$
F(\mathbf{x})=\sum_{i=1}^{m} f\left(x_{i}\right),
$$

is Schur-convex on $\mathrm{I}^{\mathrm{m}}$ iff $\mathrm{f}$ is convex on $\mathrm{I}$.

The following theorem gives weighted generalization of Majorization Theorem (see [7], [17, p. 323]).

Theorem 1.2 (Fuchs's Theorem). Let $\mathbf{x}=\left(x_{1}, \ldots, x_{m}\right), \mathbf{y}=\left(y_{1}, \ldots, y_{m}\right) \in I^{m}$ be two decreasing $m$-tuples and $\mathbf{w}=\left(w_{1}, \ldots, w_{\mathrm{m}}\right)$ be a real $\mathrm{m}$-tuple such that

$$
\sum_{i=1}^{k} w_{i} y_{i} \leqslant \sum_{i=1}^{k} w_{i} x_{i} \text { for } k=1, \ldots, m-1 ;
$$

and

$$
\sum_{i=1}^{m} w_{i} y_{i}=\sum_{i=1}^{m} w_{i} x_{i}
$$

then for every continuous convex function $\mathrm{f}: \mathrm{I} \rightarrow \mathbb{R}$, we have

$$
\sum_{i=1}^{m} w_{i} f\left(y_{i}\right) \leqslant \sum_{i=1}^{m} w_{i} f\left(x_{i}\right) .
$$

Consider the Green function $G$ defined on $[\alpha, \beta] \times[\alpha, \beta]$ by

$$
G(t, s)= \begin{cases}\frac{(t-\beta)(s-\alpha)}{\beta-\alpha}, & \alpha \leqslant s \leqslant t ; \\ \frac{(s-\beta)(t-\alpha)}{\beta-\alpha}, & t \leqslant s \leqslant \beta .\end{cases}
$$

The function $G$ is convex in $s$, it is symmetric, so it is also convex in $t$. The function $G$ is continuous in $s$ and continuous in $t$. 
Theorem 1.3 ([13]). Let $\mathrm{f}:[\alpha, \beta] \rightarrow \mathbb{R}$ be a continuous convex function on the interval $[\alpha, \beta]$ and $x=$ $\left(x_{1}, \ldots, x_{m}\right), y=\left(y_{1}, \ldots, y_{m}\right)$ and $\mathbf{w}=\left(w_{1}, \ldots, w_{m}\right)$ be m-tuples such that $x_{i}, y_{i} \in[\alpha, \beta]$ and $w_{i} \in \mathbb{R}$ $(\mathfrak{i}=1, \ldots, \mathrm{m})$ which satisfies (1.2) and also $\mathrm{G}$ is defined in (1.3).

Then the following two statements are equivalent.

(i) For every continuous convex function $f:[\alpha, \beta] \rightarrow \mathbb{R}$, it holds

$$
\sum_{i=1}^{m} w_{i} f\left(y_{i}\right) \leqslant \sum_{i=1}^{m} w_{i} f\left(x_{i}\right) .
$$

(ii) For all $\tau \in[\alpha, \beta]$, it holds

$$
\sum_{i=1}^{m} w_{i} G\left(y_{i}, \tau\right) \leqslant \sum_{i=1}^{m} w_{i} G\left(x_{i}, \tau\right) .
$$

Moreover, the statements (i) and (ii) are also equivalent if we change the sign of inequality in both inequalities, in (1.4) and in (1.5).

As mentioned in [15], the complete reference about Abel-Gontscharoff polynomial and theorem for 'two-point right focal' problem is given in [2]:

Remark 1.4. As a special choice the Abel-Gontscharoff polynomial for 'two-point right focal' interpolating polynomial for $\mathrm{n}=2$ can be given as

$$
f(z)=f(\alpha)+(z-\alpha) f^{\prime}(\beta)+\int_{\alpha}^{\beta} G_{\Omega, 2}(z, w) f^{\prime \prime}(w) d w,
$$

where $\mathrm{G}_{\Omega, 2}(z, w)$ is the Green's function for 'two-point right focal problem' given as

$$
\mathrm{G}_{1}(z, w)=\mathrm{G}_{\Omega, 2}(z, w)= \begin{cases}(\alpha-w), & \alpha \leqslant w \leqslant z \\ (\alpha-z), & z \leqslant w \leqslant \beta\end{cases}
$$

Mehmood et al. [15] introduced some new types of Green functions by keeping in view AbelGontscharoff Green's function for 'two-point right focal problem' that are:

$$
\begin{aligned}
& \mathrm{G}_{2}(z, w)= \begin{cases}(z-\beta), & \alpha \leqslant w \leqslant z, \\
(w-\beta), & z \leqslant w \leqslant \beta .\end{cases} \\
& \mathrm{G}_{3}(z, w)= \begin{cases}(z-\alpha), & \alpha \leqslant w \leqslant z, \\
(w-\alpha), & z \leqslant w \leqslant \beta .\end{cases} \\
& \mathrm{G}_{4}(z, w)= \begin{cases}(\beta-w), & \alpha \leqslant w \leqslant z, \\
(\beta-z), & z \leqslant w \leqslant \beta .\end{cases}
\end{aligned}
$$

Mehmood et al. [15] gave the following lemma, using this we obtain the new generalizations of majorization inequality.

Lemma 1.5. Let $f:[\alpha, \beta] \rightarrow \mathbb{R}$ be a twice differentiable function and $G_{p},(p=1,2,3,4)$ be the new Green functions defined above, then along with (1.6) the following identities holds:

$$
\begin{aligned}
& f(z)=f(\beta)+(z-\beta) f^{\prime}(\alpha)+\int_{\alpha}^{\beta} G_{2}(z, w) f^{\prime \prime}(w) d w, \\
& f(z)=f(\beta)-(\beta-\alpha) f^{\prime}(\alpha)+(z-\alpha) f^{\prime}(\alpha)+\int_{\alpha}^{\beta} G_{3}(z, w) f^{\prime \prime}(w) d w, \\
& f(z)=f(\alpha)-(\beta-\alpha) f^{\prime}(\alpha)-(\beta-z) f^{\prime}(\beta)+\int_{\alpha}^{\beta} G_{4}(z, w) f^{\prime \prime}(w) d w .
\end{aligned}
$$


Bernstein had proved that if all the even derivatives are at least 0 in $(a, b)$, then $f$ has an analytic continuation into the complex plane. Boas suggested to Widder that this might be proved by use of the Lidstone series. This seemed plausible because the Lidstone series, a generalization of the Taylor series, approximates a given function in the neighborhood of two points instead of one by using the even derivatives. Such series have been studied by G. J. Lidstone (1929), H. Poritsky (1932), J. M. Wittaker (1934) and others (see [3]).

Definition 1.6. Let $f \in C^{\infty}([0,1])$, then the Lidstone series has the form

$$
\sum_{k=0}^{\infty}\left(f^{(2 k)}(0) \Lambda_{k}(1-x)+f^{(2 k)}(1) \Lambda_{k}(x)\right),
$$

where, $\Lambda_{n}$ is a polynomial of degree $2 n+1$ defined by the relations

$$
\Lambda_{0}(t)=t, \quad \Lambda_{n}^{\prime \prime}(t)=\Lambda_{n-1}(t), \quad \Lambda_{n}(0)=\Lambda_{n}(1)=0, n \geqslant 1 .
$$

In [19], Widder proved the fundamental lemma:

Lemma 1.7. If $\mathrm{f} \in \mathrm{C}^{2 \mathrm{n}}([0,1])$, then

$$
f(t)=\sum_{k=0}^{n-1}\left[f^{(2 k)}(0) \Lambda_{k}(1-t)+f^{(2 k)} \Lambda_{k}(t)\right]+\int_{0}^{1} G_{n}(t, s) f^{(2 n)}(s) d s
$$

where,

$$
G_{1}(t, s)=G(t, s)= \begin{cases}(t-1) s, & s \leqslant t \\ (s-1) t, & t \leqslant s,\end{cases}
$$

is homogeneous Green's function of the differential operator $\frac{\mathrm{d}^{2}}{\mathrm{ds}^{2}}$ on $[0,1]$, and with the successive iterates of $\mathrm{G}(\mathrm{t}, \mathrm{s})$

$$
G_{n}(t, s)=\int_{0}^{1} G_{1}(t, p) G_{n-1}(p, s) d p, \quad n \geqslant 2 .
$$

The Lidstone polynomial can be expressed in terms of $G_{n}(t, s)$ as

$$
\Lambda_{\mathrm{n}}(\mathrm{t})=\int_{0}^{1} \mathrm{G}_{\mathrm{n}}(\mathrm{t}, \mathrm{s}) \mathrm{sds}
$$

When dealing with functions with different degree of smoothness, divided differences are found to be very useful.

Definition 1.8. Let $f$ be a real-valued function defined on the segment $[\alpha, \beta]$. The divided difference of order $n$ of the function $f$ at distinct points $x_{0}, \ldots, x_{n} \in[\alpha, \beta]$ is defined recursively (see [2], [17]) by

$$
f\left[x_{i}\right]=f\left(x_{i}\right), \quad(i=0, \ldots, n)
$$

and

$$
f\left[x_{0}, \ldots, x_{n}\right]=\frac{f\left[x_{1}, \ldots, x_{n}\right]-f\left[x_{0}, \ldots, x_{n-1}\right]}{x_{n}-x_{0}} .
$$

The value $f\left[x_{0}, \ldots, x_{n}\right]$ is independent of the order of the points $x_{0}, \ldots, x_{n}$.

The definition may be extended to include the case that some (or all) the points coincide. Assuming that $f^{(j-1)}(x)$ exists, we define

$$
f \underbrace{[x, \ldots, x]}_{j-\text { times }}=\frac{f^{(j-1)}(x)}{(j-1) !} .
$$

The notion of n-convexity goes back to Popoviciu [18]. We follow the definition given by Karlin [10]: 
Definition 1.9. A function $f:[\alpha, \beta] \rightarrow \mathbb{R}$ is said to be $n$-convex on $[\alpha, \beta], n \geqslant 0$ if for all choices of $(n+1)$ distinct points in $[\alpha, \beta]$, the $n$-th order divided difference of $f$ satisfies

$$
f\left[x_{0}, \ldots, x_{n}\right] \geqslant 0
$$

The Čebyšev functional has a long history and an extensive repertoire of applications in many fields including numerical quadrature, transform theory, probability and statistical problems, and special functions (see [4]). Its basic appeal stems from a desire to approximate, for example, information in the form a particular measure of the product of functions in terms of the products of the individual function measures. This inherently involves an error which may be bounded.

For two Lebesgue integrable functions $f, h:[\alpha, \beta] \rightarrow \mathbb{R}$, we consider the Čebyšev functional

$$
\Omega(f, h)=\frac{1}{\beta-\alpha} \int_{\alpha}^{\beta} f(t) h(t) d t-\frac{1}{\beta-\alpha} \int_{\alpha}^{\beta} f(t) d t \cdot \frac{1}{\beta-\alpha} \int_{\alpha}^{\beta} h(t) d t .
$$

In [5], the authors proved the following theorems.

Theorem 1.10. Let $f:[\alpha, \beta] \rightarrow \mathbb{R}$ be a Lebesgue integrable function and $h:[\alpha, \beta] \rightarrow \mathbb{R}$ be an absolutely continuous function with $(.-\alpha)(\beta-.)\left[h^{\prime}\right]^{2} \in \mathrm{L}[\alpha, \beta]$. Then we have the inequality

$$
|\Omega(f, h)| \leqslant \frac{1}{\sqrt{2}}[\Omega(f, f)]^{\frac{1}{2}} \frac{1}{\sqrt{\beta-\alpha}}\left(\int_{\alpha}^{\beta}(x-\alpha)(\beta-x)\left[h^{\prime}(x)\right]^{2} d x\right)^{\frac{1}{2}} .
$$

The constant $\frac{1}{\sqrt{2}}$ in (1.16) is the best possible.

Theorem 1.11. Assume that $h:[\alpha, \beta] \rightarrow \mathbb{R}$ is monotonic nondecreasing on $[\alpha, \beta]$ and $f:[\alpha, \beta] \rightarrow \mathbb{R}$ is absolutely continuous with $\mathrm{f}^{\prime} \in \mathrm{L}_{\infty}[\alpha, \beta]$. Then we have the inequality

$$
|\Omega(f, h)| \leqslant \frac{1}{2(\beta-\alpha)}\left\|f^{\prime}\right\|_{\infty} \int_{\alpha}^{\beta}(x-\alpha)(\beta-x) \operatorname{dh}(x) .
$$

The constant $\frac{1}{2}$ in (1.17) is the best possible.

We arrange the paper in this manner: In Section 2, we give several identities for the difference of majorization inequality by using the newly defined Green functions and Lidstone's polynomial. We obtain the generalization of majorization theorem for the class of $2 n$-convex functions for both discrete and integral case. We give bounds for identities related to the generalization of majorization inequality by using Čebyšev functional and also obtain Grüss type inequality as well as Ostrowski-type inequality for this functional.

In Section 3, we present the classical and weighted majorization theorems for the convex function $\frac{f(x)}{x}$. We give Lagrange and Cauchy type mean value theorems related to the functional which is in fact the difference of the majorization inequality. We also give n-exponential convexity which leads to exponential convexity and then log-convexity for this defined functional.

Finally, in Section 4, we consider some analytical inequalities by using our generalized results and also discuss some families of functions which enable us to construct a large families of functions that are exponentially convex and also give Stolarsky type means with their monotonicity.

\section{Generalized majorization inequality and their bounds via Green Function}

We start with the following identity that is the equivalent statements between classical weighted majorization inequality and the inequality constructed by newly defined Green functions. 
Theorem 2.1. Let $\mathbf{x}=\left(x_{1}, \ldots, x_{m}\right), \mathbf{y}=\left(y_{1}, \ldots, y_{m}\right) \in I^{m}$ be two decreasing $m$-tuples and also $\mathbf{w}=\left(w_{1}, \ldots, w_{m}\right)$ be a real $\mathrm{m}$-tuple such that satisfying (1.2) and $\mathrm{G}_{\mathrm{p}}(\mathrm{p}=1,2,3,4)$ is defined as in (1.7)-(1.10), respectively. Then the following statements are equivalent:

(i) For every continuous convex function $f:[\alpha, \beta] \rightarrow \mathbb{R}$, then

$$
\sum_{i=1}^{m} w_{i} f\left(y_{i}\right) \leqslant \sum_{i=1}^{m} w_{i} f\left(x_{i}\right)
$$

(ii) For $s \in[\alpha, \beta]$, the following inequality holds

$$
\sum_{i=1}^{m} w_{i} G_{p}\left(y_{i}, s\right) \leqslant \sum_{i=1}^{m} w_{i} G_{p}\left(x_{i}, s\right), \quad p=1,2,3,4 .
$$

Moreover, the statements (i) and (ii) are also equivalent if we change the sign of inequality in both inequalities, in (2.1) and (2.2).

Proof.

"(i) $\Rightarrow$ (ii)" Let the statement (i) holds. By fixing $p=1,2,3,4$, and as the functions $G_{p}(., s)(s \in[\alpha, \beta])$ are also continuous and convex, follows that these functions also hold inequality (2.1) for each fix p, i.e., (2.2) holds.

"(ii) $\Rightarrow$ (i)" Let $f:[\alpha, \beta] \rightarrow \mathbb{R}$ be a convex function, $f \in C^{2}([\alpha, \beta])$ and (ii) holds. Then we can represent the function $f$ in the form (1.6), (1.11), (1.12) and (1.13) for the functions $G_{p}, p=1,2,3,4$ respectively. By an easy calculation we get for all $s \in[\alpha, \beta]$,

$$
\sum_{i=1}^{m} w_{i} f\left(x_{i}\right)-\sum_{i=1}^{m} w_{i} f\left(y_{i}\right)=\int_{\alpha}^{\beta}\left(\sum_{i=1}^{m} w_{i} G_{p}\left(x_{i}, s\right)-\sum_{i=1}^{m} w_{i} G_{p}\left(y_{i}, s\right)\right) f^{\prime \prime}(s) d s \quad p=1,2,3,4 .
$$

Since $f$ is a convex function, then $f^{\prime \prime}(x) \geqslant 0$ for all $x \in[\alpha, \beta]$. So, if for every $s \in[\alpha, \beta]$ the inequality (2.2) holds for each $p=1,2,3,4$, then it follows that for every convex function $f:[\alpha, \beta] \rightarrow \mathbb{R}$, with $f \in C^{2}[\alpha, \beta]$, inequality (2.1) holds.

At the end, note that it is not necessary to demand the existence of the second derivative of the function $f([17], p .172)$. The differentiability condition can be directly eliminated by using the fact that it is possible to approximate uniformly a continuous convex functions by convex polynomials.

We present the majorization difference as in terms of Lidstone's interpolating polynomial and newly defined Green functions.

Theorem 2.2. Let $\mathrm{n} \in \mathbb{N}$ such that $\mathrm{n} \geqslant 3, \boldsymbol{x}=\left(\mathrm{x}_{1}, \ldots, \mathrm{x}_{\mathrm{m}}\right), \boldsymbol{y}=\left(\mathrm{y}_{1}, \ldots, \mathrm{y}_{\mathrm{m}}\right)$ and $\mathbf{w}=\left(w_{1}, \ldots, w_{\mathrm{m}}\right)$ be $\mathrm{m}$-tuples such that $x_{i}, y_{i} \in[\alpha, \beta]$ and $w_{i} \in \mathbb{R}(i=1, \ldots, m)$ be real $m$-tuple such that satisfying $(1.2)$ and $G_{p}(p=1,2,3,4)$ is defined as in (1.7)-(1.10), respectively. Let also $\mathrm{G}_{\mathrm{n}}$ be defined as in (1.14) and $\mathrm{f} \in \mathrm{C}^{2 \mathrm{n}}[\alpha, \beta]$, then we have the following identities for $\mathrm{p}=1,2,3,4$,

$$
\begin{aligned}
& \sum_{i=1}^{m} w_{i} f\left(x_{i}\right)-\sum_{i=1}^{m} w_{i} f\left(y_{i}\right) \\
& =\sum_{k=0}^{n-3}(\beta-\alpha)^{2 k_{f}(2 k+2)}(\alpha) \int_{\alpha}^{\beta}\left(\sum_{i=1}^{m} w_{i} G_{p}\left(x_{i}, s\right)-\sum_{i=1}^{m} w_{i} G_{p}\left(y_{i}, s\right)\right) \Lambda_{k}\left(\frac{\beta-s}{\beta-\alpha}\right) d s \\
& \quad+\sum_{k=0}^{n-3}(\beta-\alpha)^{2 k_{f}}{ }^{(2 k+2)}(\beta) \int_{\alpha}^{\beta}\left(\sum_{i=1}^{m} w_{i} G_{p}\left(x_{i}, s\right)-\sum_{i=1}^{m} w_{i} G_{p}\left(y_{i}, s\right)\right) \Lambda_{k}\left(\frac{s-\alpha}{\beta-\alpha}\right) d s
\end{aligned}
$$




$$
+(\beta-\alpha)^{2 n-1} \int_{\alpha}^{\beta} f^{(2 n)}(t)\left(\int_{\alpha}^{\beta}\left(\sum_{i=1}^{m} w_{i} G_{p}\left(x_{i}, s\right)-\sum_{i=1}^{m} w_{i} G_{p}\left(y_{i}, s\right)\right) G_{n}^{\prime \prime}\left(\frac{s-\alpha}{\beta-\alpha}, \frac{t-\alpha}{\beta-\alpha}\right) d s\right) d t
$$

where $\mathrm{G}_{n}^{\prime \prime}$ means second derivative with respect to 's'.

Proof. Fix $p=1,2,3,4$, substituting the identities (1.6), (1.11), (1.12) and (1.13) into majorization difference, we get

$$
\sum_{i=1}^{m} w_{i} f\left(x_{i}\right)-\sum_{i=1}^{m} w_{i} f\left(y_{i}\right)=\int_{\alpha}^{\beta}\left(\sum_{i=1}^{m} w_{i} G_{p}\left(x_{i}, s\right)-\sum_{i=1}^{m} w_{i} G_{p}\left(y_{i}, s\right)\right) f^{\prime \prime}(s) d s .
$$

We use Widder's Lemma for representation of function in the form

$$
\begin{aligned}
f(x)= & \sum_{k=0}^{n-1}(\beta-\alpha)^{2 k}\left[f^{(2 k)}(\alpha) \Lambda_{k}\left(\frac{\beta-x}{\beta-\alpha}\right)+f^{(2 k)}(\beta) \Lambda_{k}\left(\frac{x-\alpha}{\beta-\alpha}\right)\right] \\
& +(\beta-\alpha)^{2 n-1} \int_{\alpha}^{\beta} G_{n}\left(\frac{x-\alpha}{\beta-\alpha}, \frac{t-\alpha}{\beta-\alpha}\right) f^{(2 n)}(t) d t
\end{aligned}
$$

where, $\Lambda_{k}$ is a Lidstone polynomial.

Therefore, differentiating twice with respect to $s$, we get

$$
\begin{aligned}
f^{\prime \prime}(s)= & \sum_{k=0}^{n-3}(\beta-\alpha)^{2 k}\left[f^{(2 k+2)}(\alpha) \Lambda_{k}\left(\frac{\beta-s}{\beta-\alpha}\right)+f^{(2 k+2)}(\beta) \Lambda_{k}\left(\frac{s-\alpha}{\beta-\alpha}\right)\right] \\
& +(\beta-\alpha)^{2 n-1} \int_{\alpha}^{\beta} G_{n}^{\prime \prime}\left(\frac{s-\alpha}{\beta-\alpha}, \frac{t-\alpha}{\beta-\alpha}\right) f^{(2 n)}(t) d t .
\end{aligned}
$$

Using value of $f^{\prime \prime}(s)$ from (2.5) in (2.4), we have

$$
\begin{aligned}
& \sum_{i=1}^{m} w_{i} f\left(x_{i}\right)-\sum_{i=1}^{m} w_{i} f\left(y_{i}\right) \\
& =\sum_{k=0}^{n-3}(\beta-\alpha)^{2 k_{f}(2 k+2)}(\alpha) \int_{\alpha}^{\beta}\left(\sum_{i=1}^{m} w_{i} G_{p}\left(x_{i}, s\right)-\sum_{i=1}^{m} w_{i} G_{p}\left(y_{i}, s\right)\right) \wedge_{k}\left(\frac{\beta-s}{\beta-\alpha}\right) d s \\
& \quad+\sum_{k=0}^{n-3}(\beta-\alpha)^{2 k_{f}(2 k+2)}(\beta) \int_{\alpha}^{\beta}\left(\sum_{i=1}^{m} w_{i} G_{p}\left(x_{i}, s\right)-\sum_{i=1}^{m} w_{i} G_{p}\left(y_{i}, s\right)\right) \Lambda_{k}\left(\frac{s-\alpha}{\beta-\alpha}\right) d s \\
& \quad+(\beta-\alpha)^{2 n-1} \int_{\alpha}^{\beta}\left(\sum_{i=1}^{m} w_{i} G_{p}\left(x_{i}, s\right)-\sum_{i=1}^{m} w_{i} G_{p}\left(y_{i}, s\right)\right)\left(\int_{\alpha}^{\beta} G_{n}^{\prime \prime}\left(\frac{s-\alpha}{\beta-\alpha}, \frac{t-\alpha}{\beta-\alpha}\right) f^{(2 n)}(t) d t\right) d s,
\end{aligned}
$$

after applying Fubini's theorm we get (2.3).

Integral version of the above theorem can be stated as the following.

Theorem 2.3. Let $\mathrm{n} \in \mathbb{N}$ such that $\mathrm{n} \geqslant 3, x, y:[a, b] \rightarrow[\alpha, \beta], w:[a, b] \rightarrow \mathbb{R}$ be continuous functions such that satisfying

$$
\int_{a}^{b} w(r) y(r) d r=\int_{a}^{b} w(r) x(r) d r
$$


and $\mathrm{G}_{\mathrm{p}}(\mathrm{p}=1,2,3,4)$ is defined as in (1.7)-(1.10), respectively. Let also $\mathrm{G}_{\mathrm{n}}$ be defined as in (1.14) and $\mathrm{f} \in$ $\mathrm{C}^{2 \mathrm{n}}[\alpha, \beta]$, then we have the following identities for $\mathrm{p}=1,2,3,4$,

$$
\begin{aligned}
& \int_{a}^{b} w(r) f(x(r)) d r-\int_{a}^{b} w(r) f(y(r)) d r \\
& =\sum_{k=0}^{n-3}(\beta-\alpha)^{2 k_{f}(2 k+2)}(\alpha) \int_{a}^{b} w(r)\left[\int_{\alpha}^{\beta}\left(G_{p}(x(r), s)-G_{p}(y(r), s)\right) \Lambda_{k}\left(\frac{\beta-s}{\beta-\alpha}\right) d s\right] d r \\
& \quad+\sum_{k=0}^{n-3}(\beta-\alpha)^{2 k_{f}} f^{(2 k+2)}(\beta) \int_{a}^{b} w(r)\left[\int_{\alpha}^{\beta}\left(G_{p}(x(r), s)-G_{p}(y(r), s)\right) \Lambda_{k}\left(\frac{s-\alpha}{\beta-\alpha}\right) d s\right] d r \\
& \quad+(\beta-\alpha)^{2 n-1} \int_{\alpha}^{\beta} f^{(2 n)}(t)\left[\int_{a}^{b} w(r)\left(\int_{\alpha}^{\beta}\left(G_{p}(x(r), s)-G_{p}(y(r), s)\right) G_{n}^{\prime \prime}\left(\frac{s-\alpha}{\beta-\alpha}, \frac{t-\alpha}{\beta-\alpha}\right) d s\right) d r\right] d t
\end{aligned}
$$

where $\mathrm{G}_{\mathrm{n}}^{\prime \prime}$ means second derivative with respect to 's'.

The following theorem is the generalization of majorization theorem i.e., Fuchs's theorem.

Theorem 2.4. Let all the assumptions of Theorem 2.2 be true. If for all $s \in[\alpha, \beta]$

$$
\int_{\alpha}^{\beta}\left(\sum_{i=1}^{m} w_{i} G_{p}\left(x_{i}, s\right)-\sum_{i=1}^{m} w_{i} G_{p}\left(y_{i}, s\right)\right) G_{n}^{\prime \prime}\left(\frac{s-\alpha}{\beta-\alpha}, \frac{t-\alpha}{\beta-\alpha}\right) d s \geqslant 0,
$$

then for every $(2 \mathrm{n})$-convex function $\mathrm{f}:[\alpha, \beta] \rightarrow \mathbb{R}$, we have the following identities for $\mathrm{p}=1,2,3,4$,

$$
\begin{aligned}
& \sum_{i=1}^{m} w_{i} f\left(x_{i}\right)-\sum_{i=1}^{m} w_{i} f\left(y_{i}\right) \\
& \geqslant \sum_{k=0}^{n-3}(\beta-\alpha)^{2 k_{f}(2 k+2)}(\alpha) \int_{\alpha}^{\beta}\left(\sum_{i=1}^{m} w_{i} G_{p}\left(x_{i}, s\right)-\sum_{i=1}^{m} w_{i} G_{p}\left(y_{i}, s\right)\right) \wedge_{k}\left(\frac{\beta-s}{\beta-\alpha}\right) d s \\
& \quad+\sum_{k=0}^{n-3}(\beta-\alpha)^{2 k_{f}(2 k+2)}(\beta) \int_{\alpha}^{\beta}\left(\sum_{i=1}^{m} w_{i} G_{p}\left(x_{i}, s\right)-\sum_{i=1}^{m} w_{i} G_{p}\left(y_{i}, s\right)\right) \wedge_{k}\left(\frac{s-\alpha}{\beta-\alpha}\right) d s .
\end{aligned}
$$

If the reverse inequality in (2.7) holds, then also the reverse inequality in (2.8) holds.

Proof. Fix $p=1,2,3,4$. If the function $f$ is $2 n$-convex, without loss of generality we can assume that $f$ is $2 n$-times differentiable, we have $f^{(2 n)}(x) \geqslant 0$, for all $x \in[\alpha, \beta]$ (see [17], p. 19 and p. 293). Therefore substituting (2.7) and $f^{(2 n)}(x) \geqslant 0$ in (2.3), we get (2.8).

Integral version of the above theorem which is in fact the generalization of the weighted integral majorization theorem can be stated as follows.

Theorem 2.5. Let all the assumptions of Theorem 2.3 be true. If for all $s \in[\alpha, \beta]$

$$
\int_{a}^{b} w(r)\left(\int_{\alpha}^{\beta}\left(G_{p}(x(r), s)-G_{p}(y(r), s)\right) G_{n}^{\prime \prime}\left(\frac{s-\alpha}{\beta-\alpha}, \frac{t-\alpha}{\beta-\alpha}\right) d s\right) d r \geqslant 0,
$$

then for every $(2 \mathrm{n})$-convex function $\mathrm{f}:[\alpha, \beta] \rightarrow \mathbb{R}$, we have the following identities for $\mathrm{p}=1,2,3,4$,

$$
\int_{a}^{b} w(r) f(x(r)) d r-\int_{a}^{b} w(r) f(y(r)) d r
$$




$$
\begin{aligned}
\geqslant & \sum_{k=0}^{n-3}(\beta-\alpha)^{2 k_{f}(2 k+2)}(\alpha) \int_{a}^{b} w(r)\left[\int_{\alpha}^{\beta}\left(G_{p}(x(r), s)-G_{p}(y(r), s)\right) \Lambda_{k}\left(\frac{\beta-s}{\beta-\alpha}\right) d s\right] d r \\
& +\sum_{k=0}^{n-3}(\beta-\alpha)^{2 k_{f}}{ }^{(2 k+2)}(\beta) \int_{a}^{b} w(r)\left[\int_{\alpha}^{\beta}\left(G_{p}(x(r), s)-G_{p}(y(r), s)\right) \Lambda_{k}\left(\frac{s-\alpha}{\beta-\alpha}\right) d s\right] d r .
\end{aligned}
$$

If the reverse inequality in (2.9) holds, then also the reverse inequality in (2.10) holds.

The following theorem is majorization theorem for $2 n$-convex function.

Theorem 2.6. Let $\mathrm{n} \in \mathbb{N}$ such that $\mathrm{n} \geqslant 3$ and $\boldsymbol{x}=\left(\mathrm{x}_{1}, \ldots, \mathrm{x}_{\mathrm{m}}\right), \boldsymbol{y}=\left(\mathrm{y}_{1}, \ldots, \mathrm{y}_{\mathrm{m}}\right)$ be two decreasing real $\mathrm{m}$-tuples with $x_{i}, y_{i} \in[\alpha, \beta](i=1, \ldots, m)$ and $\mathbf{w}=\left(w_{1}, \ldots, w_{m}\right)$ be a real $\mathrm{m}$-tuple such that satisfying (1.1) and (1.2). Let also $\mathrm{G}_{\mathrm{p}}(\mathrm{p}=1,2,3,4)$ is defined as in (1.7)-(1.10), respectively.

Consider the inequality (2.8) be satisfied and let $\mathbb{F}:[\alpha, \beta] \rightarrow \mathbb{R}$ be a function defined for $p=1,2,3,4$ as

$$
\begin{aligned}
\mathbb{F}(.):= & \sum_{k=0}^{n-3}(\beta-\alpha)^{2 k} f^{(2 k+2)}(\alpha) \int_{\alpha}^{\beta} \Lambda_{k}\left(\frac{\beta-s}{\beta-\alpha}\right) G_{p}(., s) d s \\
& +\sum_{k=0}^{n-3}(\beta-\alpha)^{2 k} f^{(2 k+2)}(\beta) \int_{\alpha}^{\beta} \Lambda_{k}\left(\frac{s-\alpha}{\beta-\alpha}\right) G_{p}(., s) d s .
\end{aligned}
$$

If $\mathbb{F}$ is a convex function, then the right hand side of (2.8) is non-negative that is the following weighted majorization inequality holds

$$
\sum_{i=1}^{m} w_{i} f\left(y_{i}\right) \leqslant \sum_{i=1}^{m} w_{i} f\left(x_{i}\right) .
$$

Proof. We can easily get the equivalent form of the inequality (2.8) as

$$
\sum_{i=1}^{m} w_{i} f\left(x_{i}\right)-\sum_{i=1}^{m} w_{i} f\left(y_{i}\right) \geqslant \sum_{i=1}^{m} w_{i} \mathbb{F}\left(x_{i}\right)-\sum_{i=1}^{m} w_{i} \mathbb{F}\left(y_{i}\right) .
$$

By using majorization conditions (1.1), (1.2) and the fact that $\mathbb{F}$ is a convex function, we can apply weighted majorization inequality, which imply immediately the non-negativity of the right hand side of (2.8) and we have the inequality (2.12).

The following theorem is majorization theorem for $2 n$-convex function in integral case.

Theorem 2.7. Let $\mathrm{n} \in \mathbb{N}$ such that $\mathrm{n} \geqslant 3, x, y:[a, b] \rightarrow[\alpha, \beta]$ be decreasing and $w:[a, b] \rightarrow \mathbb{R}$ be any continuous functions satisfying

$$
\int_{a}^{v} w(r) y(r) d r \leqslant \int_{a}^{v} w(r) x(r) d r, \quad \text { for } v \in[a, b]
$$

and

$$
\int_{a}^{b} w(r) y(r) d r=\int_{a}^{b} w(r) x(r) d r .
$$

Let also $\mathrm{G}_{\mathrm{p}}(\mathrm{p}=1,2,3,4)$ be defined as in (1.7)-(1.10), respectively.

Consider the inequality (2.10) be satisfied and let $\mathbb{F}:[\alpha, \beta] \rightarrow \mathbb{R}$ be a function defined in (2.11) is a convex function, then the right hand side of (2.10) is non-negative that is the following weighted majorization inequality in integral case holds:

$$
\int_{a}^{b} w(r) f(y(r)) d r \leqslant \int_{a}^{b} w(r) f(x(r)) d r .
$$


In the next part of this section, we give the upper bounds like Grüss-type and Ostrowki-type for our generalized results.

Let $\mathbf{x}, \mathbf{y}$ be two decreasing real $\mathrm{m}$-tuples, let $\mathbf{w}=\left(w_{1}, w_{2}, \ldots, w_{\mathrm{m}}\right)$ be a real $\mathrm{m}$-tuple such that satisfying (1.2) and also $G_{n}$ and $G_{p}(p=1,2,3,4)$ defined as above, denote

$$
\Upsilon_{1}(s)=\int_{\alpha}^{\beta}\left(\sum_{i=1}^{m} w_{i} G_{p}\left(x_{i}, s\right)-\sum_{i=1}^{m} w_{i} G_{p}\left(y_{i}, s\right)\right) G_{n}^{\prime \prime}\left(\frac{s-\alpha}{\beta-\alpha}, \frac{t-\alpha}{\beta-\alpha}\right) d s,
$$

where $p=1,2,3,4, \forall s \in[\alpha, \beta]$. Similarly for $x, y:[a, b] \rightarrow[\alpha, \beta]$ and $w:[a, b] \rightarrow \mathbb{R}$ be continuous functions such that satisfying (2.6) and also $G_{n}$ and $G_{p}(p=1,2,3,4)$ defined as above, denote

$$
\Upsilon_{2}(s)=\int_{a}^{b} w(r)\left(\int_{\alpha}^{\beta}\left(G_{p}(x(r), s)-G_{p}(y(r), s)\right) G_{n}^{\prime \prime}\left(\frac{s-\alpha}{\beta-\alpha}, \frac{t-\alpha}{\beta-\alpha}\right) d s\right) d r,
$$

where $p=1,2,3,4, \forall s \in[\alpha, \beta]$.

Consider the Čebyšev functional defined as

$$
\Omega\left(\Upsilon_{u}, \Upsilon_{u}\right)=\frac{1}{\beta-\alpha} \int_{\alpha}^{\beta} \Upsilon_{u}^{2}(s) d s-\left(\frac{1}{\beta-\alpha} \int_{\alpha}^{\beta} \Upsilon_{u}(s) d s\right)^{2}, \quad u=1,2 .
$$

Theorem 2.8. Let $\mathrm{n} \in \mathbb{N}$ such that $\mathrm{n} \geqslant 3$ and $\mathrm{f}:[\alpha, \beta] \rightarrow \mathbb{R}$ be such that $\mathrm{f} \in \mathrm{C}^{2 \mathrm{n}}[\alpha, \beta]$ with $(.-\alpha)(\beta-$ .) $\left[\mathrm{f}^{(2 \mathrm{n}+1)}\right]^{2} \in \mathrm{L}[\alpha, \beta]$, and also $x, y$ be two decreasing real m-tuples such that $x_{i}, y_{i} \in[\alpha, \beta]$ and $w_{i} \in \mathbb{R}$ $(i=1,2, \ldots, m)$ satisfying (1.2). Let also the functions $G_{p}(p=1,2,3,4)$ be defined as in (1.7)-(1.10) respectively and $\Upsilon_{1}$ be defined in (2.13). Then the remainder $\mathbb{R} \mathbb{E} M(f ; \alpha, \beta)$ defined for $p=1,2,3,4$ as

$$
\begin{aligned}
\mathbb{R E M}(f ; \alpha, \beta)= & \sum_{i=1}^{m} w_{i} f\left(x_{i}\right)-\sum_{i=1}^{m} w_{i} f\left(y_{i}\right) \\
& -\sum_{k=0}^{n-3}(\beta-\alpha)^{2 k_{f}(2 k+2)}(\alpha) \int_{\alpha}^{\beta}\left(\sum_{i=1}^{m} w_{i} G_{p}\left(x_{i}, s\right)-\sum_{i=1}^{m} w_{i} G_{p}\left(y_{i}, s\right)\right) \Lambda_{k}\left(\frac{\beta-s}{\beta-\alpha}\right) d s \\
& -\sum_{k=0}^{n-3}(\beta-\alpha)^{2 k_{f}(2 k+2)}(\beta) \int_{\alpha}^{\beta}\left(\sum_{i=1}^{m} w_{i} G_{p}\left(x_{i}, s\right)-\sum_{i=1}^{m} w_{i} G_{p}\left(y_{i}, s\right)\right) \Lambda_{k}\left(\frac{s-\alpha}{\beta-\alpha}\right) d s \\
& -(\beta-\alpha)^{2 n-2}\left(f^{(2 n-1)}(\beta)-f^{(2 n-1)}(\alpha)\right) \int_{\alpha}^{\beta} \Upsilon_{1}(t) d t
\end{aligned}
$$

satisfies the estimation

$$
|\operatorname{REM}(f ; \alpha, \beta)| \leqslant \frac{(\beta-\alpha)^{2 n-\frac{1}{2}}}{\sqrt{2}}\left[\Omega\left(\Upsilon_{1}, \Upsilon_{1}\right)\right]^{\frac{1}{2}}\left|\int_{\alpha}^{\beta}(t-\alpha)(\beta-t)\left[f^{(2 n+1)}(t)\right]^{2} d t\right|^{\frac{1}{2}}
$$

Proof. Comparing (2.15) and (2.3) we have

$$
\mathbb{R E M}(f ; \alpha, \beta)=(\beta-\alpha) \Omega\left(\Upsilon_{1}, f^{(2 n)}\right) .
$$

Applying Theorem 1.10 on the functions $\Upsilon$ and $f^{(2 n)}$, we obtain (2.16).

Integral case of the above theorem can be given:

Theorem 2.9. Let $\mathrm{n} \in \mathbb{N}$ such that $\mathrm{n} \geqslant 3$ and $\mathrm{f}:[\alpha, \beta] \rightarrow \mathbb{R}$ be such that $f \in \mathrm{C}^{2 \mathrm{n}}[\alpha, \beta]$ with $(.-\alpha)(\beta-$ .) $\left[f^{(2 n+1)}\right]^{2} \in L[\alpha, \beta]$, and $x, y:[a, b] \rightarrow[\alpha, \beta], w:[a, b] \rightarrow \mathbb{R}$ be continuous functions satisfying (2.6). Let also 
the functions $G_{p}(p=1,2,3,4)$ be defined as in (1.7)-(1.10) respectively and $\Upsilon_{2}$ be defined as in (2.14). Then the remainder $\widetilde{\mathbb{R E M}}(f ; \alpha, \beta)$ defined for $p=1,2,3,4$ as

$$
\begin{aligned}
\widetilde{\mathbb{R E M}}(f ; \alpha, \beta)= & \int_{a}^{b} w(r) f(x(r)) d r-\int_{a}^{b} w(r) f(y(r)) d r \\
& -\sum_{k=0}^{n-3}(\beta-\alpha)^{2 k_{f}(2 k+2)}(\alpha) \int_{a}^{b} w(r)\left[\int_{\alpha}^{\beta}\left(G_{p}(x(r), s)-G_{p}(y(r), s)\right) \Lambda_{k}\left(\frac{\beta-s}{\beta-\alpha}\right) d s\right] d r \\
& -\sum_{k=0}^{n-3}(\beta-\alpha)^{2 k_{f}(2 k+2)}(\beta) \int_{a}^{b} w(r)\left[\int_{\alpha}^{\beta}\left(G_{p}(x(r), s)-G_{p}(y(r), s)\right) \Lambda_{k}\left(\frac{s-\alpha}{\beta-\alpha}\right) d s\right] d r \\
& -(\beta-\alpha)^{2 n-2}\left(f^{(2 n-1)}(\beta)-f^{(2 n-1)}(\alpha)\right) \int_{\alpha}^{\beta} \Upsilon_{2}(s) d s,
\end{aligned}
$$

satisfies the estimation

$$
|\widetilde{\mathbb{R E M}}(f ; \alpha, \beta)| \leqslant \frac{(\beta-\alpha)^{2 n-\frac{1}{2}}}{\sqrt{2}}\left[\Omega\left(\Upsilon_{2}, \Upsilon_{2}\right)\right]^{\frac{1}{2}}\left|\int_{\alpha}^{\beta}(t-\alpha)(\beta-t)\left[f^{(2 n+1)}(t)\right]^{2} d t\right|^{\frac{1}{2}}
$$

Using Theorem 1.11, we obtain the following Grüss type inequality.

Theorem 2.10. Let $\mathrm{n} \in \mathbb{N}$ such that $\mathrm{n} \geqslant 3$ and $\mathrm{f}:[\alpha, \beta] \rightarrow \mathbb{R}$ be such that $\mathrm{f} \in \mathrm{C}^{2 \mathrm{n}}[\alpha, \beta]$ and also $\mathrm{f}^{(2 \mathrm{n}+1)} \geqslant 0$ on $[\alpha, \beta]$. Let the function $\Upsilon_{1}$ be defined as in (2.13). Then the remainder $\mathbb{R} \mathbb{E} \mathbb{M}(f ; \alpha, \beta)$ defined by (2.15) satisfies the estimation

$$
|\mathbb{R} \mathbb{E M}(f ; \alpha, \beta)| \leqslant(\beta-\alpha)^{2 n-1}\left\|\Upsilon_{1}^{\prime}\right\|_{\infty}\left\{\frac{f^{(2 n-1)}(\beta)+f^{(2 n-1)}(\alpha)}{2}-\frac{f^{(2 n-2)}(\beta)-f^{(2 n-2)}(\alpha)}{\beta-\alpha}\right\} .
$$

Proof. Since $\mathbb{R} \mathbb{E M}(f ; \alpha, \beta)=(\beta-\alpha)^{2 \mathfrak{n}} \Omega\left(\Upsilon_{1}, f^{(2 n)}\right)$, applying Theorem 1.11 on the functions $\Upsilon_{1}$ and $f^{(2 n)}$, we get (2.18).

Integral version of the above theorem can be given as follows.

Theorem 2.11. Let $\mathrm{n} \in \mathbb{N}$ such that $\mathrm{n} \geqslant 3$ and $\mathrm{f}:[\alpha, \beta] \rightarrow \mathbb{R}$ be such that $\mathrm{f} \in \mathrm{C}^{2 \mathrm{n}}[\alpha, \beta]$ and also $\mathrm{f}^{(2 \mathrm{n}+1)} \geqslant 0$ on $[\alpha, \beta]$. Let also the function $\Upsilon_{2}$ be defined as in (2.14). Then the remainder $\widetilde{\mathbb{R E M}}(\mathrm{f} ; \alpha, \beta)$ defined by (2.17) satisfies the estimation

$$
|\widetilde{\mathbb{R E M}}(f ; \alpha, \beta)| \leqslant(\beta-\alpha)^{2 n-1}\left\|\gamma_{2}^{\prime}\right\|_{\infty}\left\{\frac{f^{(2 n-1)}(\beta)+f^{(2 n-1)}(\alpha)}{2}-\frac{f^{(2 n-2)}(\beta)-f^{(2 n-2)}(\alpha)}{\beta-\alpha}\right\} .
$$

We give the Ostrowski-type inequality related to our generalized result.

Theorem 2.12. Suppose that all the assumptions of Theorem 2.2 hold. Assume $(u, v)$ is a pair of conjugate exponents, that is $1 \leqslant u, v \leqslant \infty, \frac{1}{u}+\frac{1}{v}=1$. Let $\left|\mathrm{f}^{(2 \mathrm{n})}\right|^{\mathrm{u}}:[\alpha, \beta] \rightarrow \mathbb{R}$ be an R-integrable function for some $\mathrm{n} \in \mathbb{N}$. Then we have the following identities for $\mathrm{p}=1,2,3,4$

$$
\begin{aligned}
& \mid \sum_{i=1}^{m} w_{i} f\left(x_{i}\right)-\sum_{i=1}^{m} w_{i} f\left(y_{i}\right) \\
& \quad-\sum_{k=0}^{n-3}(\beta-\alpha)^{2 k_{f}(2 k+2)}(\alpha) \int_{\alpha}^{\beta}\left(\sum_{i=1}^{m} w_{i} G_{p}\left(x_{i}, s\right)-\sum_{i=1}^{m} w_{i} G_{p}\left(y_{i}, s\right)\right) \Lambda_{k}\left(\frac{\beta-s}{\beta-\alpha}\right) d s \\
& \quad-\sum_{k=0}^{n-3}(\beta-\alpha)^{2 k_{f}(2 k+2)}(\beta) \int_{\alpha}^{\beta}\left(\sum_{i=1}^{m} w_{i} G_{p}\left(x_{i}, s\right)-\sum_{i=1}^{m} w_{i} G_{p}\left(y_{i}, s\right)\right) \Lambda_{k}\left(\frac{s-\alpha}{\beta-\alpha}\right) d s \mid \\
& \quad \leqslant(\beta-\alpha)^{2 n-1}\left\|f^{(2 n)}\right\|_{u}\left(\int_{\alpha}^{\beta}\left|\int_{\alpha}^{\beta}\left(\sum_{i=1}^{m} w_{i} G_{p}\left(x_{i}, s\right)-\sum_{i=1}^{m} w_{i} G_{p}\left(y_{i}, s\right)\right) G_{n}^{\prime \prime}\left(\frac{s-\alpha}{\beta-\alpha}, \frac{t-\alpha}{\beta-\alpha}\right) d s\right|^{v} d t\right)^{\frac{1}{v}} .
\end{aligned}
$$

The constant on the right-hand side of (2.19) is sharp for $1<u \leqslant \infty$ and the best possible for $u=1$. 
Proof. Let us denote

$$
\Psi(t)=(\beta-\alpha)^{2 n-1} \int_{\alpha}^{\beta}\left(\sum_{i=1}^{m} w_{i} G_{p}\left(x_{i}, s\right)-\sum_{i=1}^{m} w_{i} G_{p}\left(y_{i}, s\right)\right) G_{n}^{\prime \prime}\left(\frac{s-\alpha}{\beta-\alpha}, \frac{t-\alpha}{\beta-\alpha}\right) d s, \text { for } p=1,2,3,4 .
$$

Using the identity (2.3) and applying Hölder's inequality, we obtain

$$
\begin{aligned}
& \mid \sum_{i=1}^{m} w_{i} f\left(x_{i}\right)-\sum_{i=1}^{m} w_{i} f\left(y_{i}\right) \\
& \quad-\sum_{k=0}^{n-3}(\beta-\alpha)^{2 k_{f}(2 k+2)}(\alpha) \int_{\alpha}^{\beta}\left(\sum_{i=1}^{m} w_{i} G_{p}\left(x_{i}, s\right)-\sum_{i=1}^{m} w_{i} G_{p}\left(y_{i}, s\right)\right) \wedge_{k}\left(\frac{\beta-s}{\beta-\alpha}\right) d s \\
& \quad-\sum_{k=0}^{n-3}(\beta-\alpha)^{2 k_{f}(2 k+2)}(\beta) \int_{\alpha}^{\beta}\left(\sum_{i=1}^{m} w_{i} G_{p}\left(x_{i}, s\right)-\sum_{i=1}^{m} w_{i} G_{p}\left(y_{i}, s\right)\right) \wedge_{k}\left(\frac{s-\alpha}{\beta-\alpha}\right) d s \mid \\
& =\left|\int_{\alpha}^{\beta} \Psi(t) f^{(2 n)}(t) d t\right| \leqslant\left\|f^{(2 n)}\right\|_{u}\left(\int_{\alpha}^{\beta}|\Psi(t)|^{v} d t\right)^{\frac{1}{v}} .
\end{aligned}
$$

The proof of the sharpness of the constant $\left(\int_{\alpha}^{\beta}|\Psi(t)|^{v} d t\right)^{\frac{1}{v}}$ is analog to one in proof of Theorem 11 in [1].

Integral version of the above theorem can be stated as follows.

Theorem 2.13. Suppose that all the assumptions of Theorem 2.3 hold. Assume $(u, v)$ is a pair of conjugate exponents, that is $1 \leqslant u, v \leqslant \infty, \frac{1}{u}+\frac{1}{v}=1$. Let $\left|\mathrm{f}^{(2 \mathrm{n})}\right|^{\mathfrak{u}}:[\alpha, \beta] \rightarrow \mathbb{R}$ be an R-integrable function for some $\mathrm{n} \in \mathbb{N}$. Then we have the following identities for $\mathrm{p}=1,2,3,4$

$$
\begin{aligned}
& \mid \int_{a}^{b} w(r) f(x(r)) d r-\int_{a}^{b} w(r) f(y(r)) d r \\
& \quad-\sum_{k=0}^{n-3}(\beta-\alpha)^{2 k_{f}(2 k+2)}(\alpha) \int_{a}^{b} w(r)\left[\int_{\alpha}^{\beta}\left(G_{p}(x(r), s)-G_{p}(y(r), s)\right) \Lambda_{k}\left(\frac{\beta-s}{\beta-\alpha}\right) d s\right] d r \\
& \quad-\sum_{k=0}^{n-3}(\beta-\alpha)^{2 k_{f}}{ }^{(2 k+2)}(\beta) \int_{a}^{b} w(r)\left[\int_{\alpha}^{\beta}\left(G_{p}(x(r), s)-G_{p}(y(r), s)\right) \Lambda_{k}\left(\frac{s-\alpha}{\beta-\alpha}\right) d s\right] d r \mid \\
& \leqslant(\beta-\alpha)^{2 n-1}\left\|f^{(2 n)}\right\|_{u}\left(\int_{\alpha}^{\beta}\left|\int_{a}^{b} w(r)\left(\int_{\alpha}^{\beta}\left(G_{p}(x(r), s)-G_{p}(y(r), s)\right) G_{n}^{\prime \prime}\left(\frac{s-\alpha}{\beta-\alpha}, \frac{t-\alpha}{\beta-\alpha}\right) d s\right) d r\right|^{v} d t\right)^{\frac{1}{v}} .
\end{aligned}
$$

The constant on the right-hand side of (2.20) is sharp for $1<u \leqslant \infty$ and the best possible for $\mathrm{u}=1$.

Motivated by the inequality (2.8) and (2.10), we define functionals $\Theta_{1}(f)$ and $\Theta_{2}(f)$ by

$$
\begin{aligned}
\Theta_{1}(f)= & \sum_{i=1}^{m} w_{i} f\left(x_{i}\right)-\sum_{i=1}^{m} w_{i} f\left(y_{i}\right) \\
& -\sum_{k=0}^{n-3}(\beta-\alpha)^{2 k} f^{(2 k+2)}(\alpha) \int_{\alpha}^{\beta}\left(\sum_{i=1}^{m} w_{i} G_{p}\left(x_{i}, s\right)-\sum_{i=1}^{m} w_{i} G_{p}\left(y_{i}, s\right)\right) \wedge_{k}\left(\frac{\beta-s}{\beta-\alpha}\right) d s \\
& -\sum_{k=0}^{n-3}(\beta-\alpha)^{2 k_{f}}{ }^{(2 k+2)}(\beta) \int_{\alpha}^{\beta}\left(\sum_{i=1}^{m} w_{i} G_{p}\left(x_{i}, s\right)-\sum_{i=1}^{m} w_{i} G_{p}\left(y_{i}, s\right)\right) \wedge_{k}\left(\frac{s-\alpha}{\beta-\alpha}\right) d s,
\end{aligned}
$$




$$
\begin{aligned}
\Theta_{2}(f)= & \int_{a}^{b} w(r) f(x(r)) d r-\int_{a}^{b} w(r) f(y(r)) d r \\
& -\sum_{k=0}^{n-3}(\beta-\alpha)^{2 k_{f}} f^{(2 k+2)}(\alpha) \int_{a}^{b} w(r)\left[\int_{\alpha}^{\beta}\left(G_{p}(x(r), s)-G_{p}(y(r), s)\right) \Lambda_{k}\left(\frac{\beta-s}{\beta-\alpha}\right) d s\right] d r \\
& -\sum_{k=0}^{n-3}(\beta-\alpha)^{2 k_{f}}{ }^{(2 k+2)}(\beta) \int_{a}^{b} w(r)\left[\int_{\alpha}^{\beta}\left(G_{p}(x(r), s)-G_{p}(y(r), s)\right) \Lambda_{k}\left(\frac{s-\alpha}{\beta-\alpha}\right) d s\right] d r .
\end{aligned}
$$

Lagrange and Cauchy type mean value theorems related to defined functionals are given in the following theorems.

Theorem 2.14. Let $f:[\alpha, \beta] \rightarrow \mathbb{R}$ be such that $f \in C^{2 n}[\alpha, \beta]$. If the inequalities in $(2.7)(i=1)$, (2.9) $(i=2)$ hold, then there exist $\xi_{i} \in[\alpha, \beta]$ such that

$$
\Theta_{i}(f)=f^{(2 n)}(\xi) \Theta_{i}(\eta), \quad i=1,2,
$$

where $\eta(x)=\frac{x^{2 n}}{(2 n) !}$.

Proof. Similar to the proof of Theorem 7 in [3].

Theorem 2.15. Let $f, g:[\alpha, \beta] \rightarrow \mathbb{R}$ be such that $f, g \in C^{2 n}[\alpha, \beta]$. If the inequalities in $(2.7)(i=1)$, (2.9) $(i=2)$ hold, then there exist $\xi_{i} \in[\alpha, \beta]$ such that

$$
\frac{\Theta_{i}(f)}{\Theta_{i}(g)}=\frac{f^{(2 n)}(\xi)}{g^{(2 n)}(\xi)}, i=1,2,
$$

provided that the denominators are not zero.

Proof. Similar to the proof of Corollary 12 in [3].

\section{Further Generalized Results of Majorization Inequality via convex function $\frac{f(x)}{x}$}

For example, in the papers [11] and [12] we gave the results about majorization in the form of $n$ exponentially, exponentially and logarithmically convex functions as well as generalized Cauchy mean value theorems for class of convex functions $f$, but now we present these results for the class of convex functions $f(x) / x$ and also an important thing is to construct examples for such type of results. So first we give the classical results for convex function $f(x) / x$ and then make functionals for obtaining n-exponentially, exponentially and logarithmically convex functions.

Theorem 3.1. Let $I_{+} \subset \mathbb{R}$ be an interval and $\mathbf{x}=\left(x_{1}, \ldots, x_{m}\right), \mathbf{y}=\left(y_{1}, \ldots, y_{m}\right) \in I_{+}^{m}$. Let $f: I_{+} \rightarrow \mathbb{R}$ be continuous function, then a function $\mathbb{F}: \mathrm{I}_{+}^{\mathrm{m}} \rightarrow \mathbb{R}$, defined by

$$
\mathbb{F}(\mathbf{x})=\sum_{i=1}^{m} \frac{f\left(x_{i}\right)}{x_{i}},
$$

is Schur-convex on $\mathrm{I}_{+}^{\mathrm{m}}$ iff $\frac{\mathrm{f}(\mathrm{x})}{\mathrm{x}}$ is convex on $\mathrm{I}_{+}$.

Proof. In this proof we use Abel's transformation. Without loss of generality, assume that $x_{i} \neq y_{i}$, define

$$
\Delta_{i}=\frac{\frac{f\left(y_{i}\right)}{y_{i}}-\frac{f\left(x_{i}\right)}{x_{i}}}{y_{i}-x_{i}}, \quad i=(1, \ldots, m) .
$$

Since the function $\frac{f(x)}{x}$ is convex, and using $x_{[i]}$ 's and $y_{[i]}$ 's defined above, we get that $\Delta_{i+1} \leqslant \Delta_{i}$, for $(i=1,2, \ldots, m)$, which means that $\Delta_{i}$ is decreasing. The proof follows from see pages 323-324 in [17]. 
The weighted version of the above theorem is stated as follows.

Theorem 3.2. Let $\mathbf{x}, \mathbf{y}$ be two decreasing positive m-tuples, let $\mathbf{w}=\left(w_{1}, w_{2}, \ldots, w_{m}\right)$ be a real $n$-tuple such that

$$
\sum_{i=1}^{k} w_{i} y_{i} \leqslant \sum_{i=1}^{k} w_{i} x_{i} \text { for } k=1, \ldots, m-1,
$$

and

$$
\sum_{i=1}^{m} w_{i} y_{i}=\sum_{i=1}^{m} w_{i} x_{i}
$$

Then for every convex function $\frac{\mathrm{f}(\mathrm{x})}{\mathrm{x}}: \mathrm{I}_{+} \rightarrow \mathrm{R}$, we have

$$
\sum_{i=1}^{m} w_{i} \frac{f\left(y_{i}\right)}{y_{i}} \leqslant \sum_{i=1}^{m} w_{i} \frac{f\left(x_{i}\right)}{x_{i}} .
$$

Proof. The proof is similar to the Theorem 3.1.

Motivated by the inequalities (3.1) and (3.4) that are linear in $f$, we define the linear functionals under the assumptions of Theorem 3.1 and Theorem 3.2:

$$
\Lambda_{1}(\mathbf{x}, \mathbf{y}, f)=\sum_{i=1}^{m} \frac{f\left(x_{i}\right)}{x_{i}}-\sum_{i=1}^{m} \frac{f\left(y_{i}\right)}{y_{i}},
$$

and

$$
\Lambda_{2}(\mathbf{x}, \mathbf{y}, \mathbf{f})=\sum_{i=1}^{m} w_{i} \frac{f\left(x_{i}\right)}{x_{i}}-\sum_{i=1}^{m} w_{i} \frac{f\left(y_{i}\right)}{y_{i}} .
$$

Under the assumptions of Theorem 3.1 and Theorem 3.2, it holds $\Lambda_{l}(f) \geqslant 0, l=1,2$, for all convex functions $\frac{f(x)}{x}$.

The following Lemma is given in [14]:

Lemma 3.3. Let $\mathrm{f} \in \mathrm{C}^{2}(\mathrm{I})$ for an interval $\mathrm{I} \subset \mathbb{R} \backslash\{0\}$ and consider $\mathrm{m}, \mathrm{M} \in \mathbb{R}$ such that

$$
m \leqslant \frac{x^{2} f^{\prime \prime}(x)-2 x f^{\prime}(x)+2 f(x)}{x^{3}} \leqslant M
$$

Also, let $\mathrm{f}_{1}, \mathrm{f}_{2}$ be real valued functions defined on $\mathrm{I}$ as follows

$$
\begin{aligned}
& f_{1}(x)=M \frac{x^{3}}{2}-f(x), \\
& f_{2}(x)=f(x)-m \frac{x^{3}}{2} .
\end{aligned}
$$

Then $\frac{f_{1}(x)}{x}$ and $\frac{f_{2}(x)}{x}$ are convex.

Lagrange and Cauchy type mean value theorems related to defined functionals are given in the following theorems:

Theorem 3.4. Let $\mathbf{x}, \mathbf{y}$ be two real m-tuples.

$\cdot \mathbf{x} \succ \mathbf{y}$ for $\mathbf{l}=1$, 
- $\mathbf{x}, \mathbf{y}$ be decreasing and let $\mathbf{w}=\left(w_{1}, w_{2}, \ldots, w_{m}\right)$ be a real $\mathrm{m}$-tuple such that satisfying (3.2) and (3.3) for $l=2$.

Let $[\alpha, \beta] \subset \mathbb{R}^{+}$and $f \in C^{2}([\alpha, \beta])$ then there exists $\xi_{l} \in[\alpha, \beta]$ such that

$$
\Lambda_{l}(\mathbf{x}, \mathbf{y}, \mathbf{f})=\frac{\xi_{l}^{2} f^{\prime \prime}\left(\xi_{l}\right)-2 \xi_{l} f^{\prime}\left(\xi_{l}\right)+2 f\left(\xi_{l}\right)}{2 \xi_{l}^{3}} \Lambda_{l}\left(\mathbf{x}, \mathbf{y}, x^{3}\right), \quad l=1,2 .
$$

Proof. Fix $l=1,2$ (see Theorem 2.8 in [14]), by convexity of $f_{1}(x)$ and $f_{2}(x)$ from Lemma 3.3 therefore (3.1) changes to

$$
\Lambda_{l}(\mathbf{x}, \mathbf{y}, \mathbf{f}) \leqslant \frac{M}{2} \Lambda_{l}\left(\mathbf{x}, \mathbf{y}, x^{3}\right)
$$

and

$$
\frac{m}{2} \Lambda_{l}\left(\mathbf{x}, \mathbf{y}, x^{3}\right) \leqslant \Lambda_{l}(\mathbf{x}, \mathbf{y}, \mathbf{f})
$$

Since $\Lambda_{l}\left(\mathbf{x}, \mathbf{y}, x^{3}\right) \neq 0$, so from (3.8) and (3.9) we have

$$
m \leqslant \frac{2 \Lambda_{l}(x, y, f)}{\Lambda_{l}\left(x, y, x^{3}\right)} \leqslant M
$$

Therefore we get the required result by using Lemma 3.3.

Theorem 3.5. Let $\mathbf{x}, \mathbf{y}$ be two real m-tuples.

$\cdot \mathbf{x} \succ \mathbf{y}$ for $\mathbf{l}=1$,

- $\mathbf{x}, \mathbf{y}$ be decreasing and let $\mathbf{w}=\left(w_{1}, w_{2}, \ldots, w_{m}\right)$ be a real $\mathrm{m}$-tuple such that satisfying (3.2) and (3.3) for $l=2$.

Let $[\alpha, \beta] \subset \mathbb{R}^{+}$and $f, g \in C^{2}([\alpha, \beta])$, then there exists $\xi_{l} \in[\alpha, \beta]$ such that

$$
\frac{\Lambda_{l}(\mathbf{x}, \mathbf{y}, \mathbf{f})}{\Lambda_{l}(\mathbf{x}, \mathbf{y}, g)}=\frac{\xi_{l}^{2} f^{\prime \prime}\left(\xi_{l}\right)-2 \xi_{l} f^{\prime}\left(\xi_{l}\right)+2 f\left(\xi_{l}\right)}{\xi_{l}^{2} g^{\prime \prime}\left(\xi_{l}\right)-2 \xi_{l} g^{\prime}\left(\xi_{l}\right)+2 g\left(\xi_{l}\right)}, \quad l=1,2,
$$

provided that denominators are non-zero.

Proof. Fix $l=1,2$ (Theorem 2.9 of [14]), define $h \in C^{2}([\alpha, \beta])$ in the way that

$$
h=c_{1} f-c_{2} g, \quad \text { where } \quad c_{1}=\Lambda_{1}(x, y, g) \text { and } c_{2}=\Lambda_{l}(\mathbf{x}, \mathbf{y}, f) .
$$

Now using (3.7) with $f=h$, we have

$$
\left(c_{1}\left(\frac{\xi^{2} f^{\prime \prime}(\xi)-2 \xi f^{\prime}(\xi)+2 f(\xi)}{2 \xi^{3}}\right)-c_{2}\left(\frac{\xi^{2} g^{\prime \prime}(\xi)-2 \xi g^{\prime}(\xi)+2 g(\xi)}{2 \xi^{3}}\right)\right) \Lambda_{l}\left(\mathbf{x}, \mathbf{y}, \chi^{3}\right)=0 .
$$

Since $\Lambda_{l}\left(\mathbf{x}, \mathbf{y}, x^{3}\right) \neq 0$, therefore we get the required result.

Now, we give the notion and some facts of exponentially convex functions (see [9]).

Definition 3.6. A function $\mathrm{f}: \mathrm{I} \rightarrow \mathbb{R}$ is n-exponentially convex in the Jensen sense on I if

$$
\sum_{k, l=1}^{n} \alpha_{k} \alpha_{l} f\left(\frac{x_{k}+x_{l}}{2}\right) \geqslant 0
$$

holds for $\alpha_{k} \in \mathbb{R}$ and $x_{k} \in I, k=1,2, \ldots, n$. 
A function $\mathrm{f}: \mathrm{I} \rightarrow \mathbb{R}$ is n-exponentially convex on $\mathrm{I}$ if it is $n$-exponentially convex in the Jensen sense and continuous on I. It is clear that 1-exponentially convex functions in the Jensen sense are in fact non-negative functions. Also, $n$-exponentially convex functions in the Jensen sense are m-exponentially convex in the Jensen sense for every $m \in \mathbb{N}, m \leqslant n$.

Proposition 3.7. If $\mathrm{f}: \mathrm{I} \rightarrow \mathbb{R}$ is an $\mathrm{n}$-exponentially convex in the Jensen sense, then the matrix $\left[\phi\left(\frac{\mathrm{x}_{\mathrm{k}}+\mathrm{x}_{\mathrm{l}}}{2}\right)\right]_{\mathrm{k}, \mathrm{l}=1}^{\mathrm{m}}$ is a positive semi-definite matrix for all $\mathrm{m} \in \mathbb{N}, \mathrm{m} \leqslant \mathrm{n}$. Particularly,

$$
\operatorname{det}\left[f\left(\frac{x_{k}+x_{l}}{2}\right)\right]_{k, l=1}^{m} \geqslant 0
$$

for all $\mathrm{m} \in \mathbb{N}, \mathrm{m}=1,2, \ldots, \mathrm{n}$.

A function $f: I \rightarrow \mathbb{R}$ is exponentially convex in the Jensen sense on $I$ if it is $n$-exponentially convex in the Jensen sense for all $n \in \mathbb{N}$. A function $f: I \rightarrow \mathbb{R}$ is exponentially convex if it is exponentially convex in the Jensen sense and continuous.

Remark 3.8. It is easy to show that $\mathrm{f}: \mathrm{I} \rightarrow \mathbb{R}$ is log-convex in the Jensen sense if and only if

$$
\alpha^{2} f(x)+2 \alpha \beta f\left(\frac{x+y}{2}\right)+\beta^{2} f(y) \geqslant 0
$$

holds for every $\alpha, \beta \in \mathbb{R}$ and $x, y \in$ I. It follows that a function is log-convex in the Jensen-sense if and only if it is 2-exponentially convex in the Jensen sense.

Remark 3.9. Also, using basic convexity theory it follows that a function is log-convex if and only if it is 2-exponentially convex.

Corollary 3.10. If $\mathrm{f}: \mathrm{I} \rightarrow(0, \infty)$ is an exponentially convex function, then $\mathrm{f}$ is a log-convex function that is

$$
f(\lambda x+(1-\lambda) y) \leqslant f^{\lambda}(x) f^{1-\lambda}(y), \text { for all } x, y \in I, \lambda \in[0,1]
$$

In order to obtain results regarding the exponential convexity, we define the families of functions as follows.

For every choice of $t+1$ mutually different points $z_{0}, \ldots, z_{t} \in[\alpha, \beta]$ we define

- $\mathbb{F}_{1}=\left\{\mathrm{f}_{v}:[\alpha, \beta] \rightarrow \mathrm{R}: v \in \mathrm{J}\right.$ and $\mathrm{v} \mapsto\left[\mathrm{z}_{0}, \ldots, \mathrm{z}_{\mathrm{t}}, \frac{\mathrm{f}_{\mathrm{v}}(\mathrm{x})}{\mathrm{x}}\right]$ is $\mathrm{n}$-exponentially convex in the Jensen sense on $\mathrm{J}\}$

- $\mathbb{F}_{2}=\left\{f_{v}:[\alpha, \beta] \rightarrow R: v \in J\right.$ and $\mathrm{v} \mapsto\left[\mathrm{z}_{0}, \ldots, \mathrm{z}_{\mathrm{t}}, \frac{\mathrm{f}_{\mathrm{v}}(\mathrm{x})}{\mathrm{x}}\right]$ is exponentially convex in the Jensen sense on J\}

- $\mathbb{F}_{3}=\left\{\mathrm{f}_{v}:[\alpha, \beta] \rightarrow \mathrm{R}: v \in \mathrm{J}\right.$ and $\mathrm{v} \mapsto\left[\mathrm{z}_{0}, \ldots, \mathrm{z}_{\mathrm{t}}, \frac{\mathrm{f}_{\mathrm{v}}(\mathrm{x})}{\mathrm{x}}\right]$ is 2-exponentially convex in the Jensen sense on $\mathrm{J}\}$

Theorem 3.11. Let $\Lambda_{l}(l=1,2)$ be the linear functionals defined by (3.5) and (3.6) associated with family $\mathbb{F}_{1}$. Then the following statements hold:

(i) The function $v \mapsto \Lambda_{l}\left(f_{v}\right)$ is an n-exponentially convex function in the Jensen sense on $\mathrm{J}$ and the matrix $\left[\Lambda_{l}\left(f_{\frac{v_{i}+v_{j}}{2}}\right)\right]_{i, j=1}^{p}$ is a positive semi-definite. Particularly

$$
\operatorname{det}\left[\Lambda_{l}\left(f_{\frac{v_{i}+v_{j}}{2}}\right)\right]_{i, j=1}^{p} \geqslant 0
$$

holds for all $p \in \mathbb{N}, p \leqslant n, v_{1}, \ldots, v_{p} \in J$. 
(ii) If the function $v \mapsto \Lambda_{l}\left(f_{v}\right)$ is continuous on $\mathrm{J}$, then it is n-exponentially convex function on $\mathrm{J}$.

Proof. (i) For fix $l=1,2, \vartheta_{i} \in \mathbb{R}$ and $v_{i} \in J, i=1, \ldots, n$ we define the function

$$
\delta(x)=\sum_{i, j=1}^{n} \vartheta_{i} \vartheta_{j} \frac{f_{v_{i}+v_{j}}(x)}{x} .
$$

Using the assumption we have

$$
\left[z_{0}, \ldots, z_{\mathrm{t}}, \delta\right]=\sum_{i, j=1}^{n} \vartheta_{i} \vartheta_{j}\left[z_{0}, \ldots, z_{\mathrm{t}}, \frac{f_{v_{i}+v_{j}}(x)}{x}\right] \geqslant 0,
$$

which in turn implies the required results (see Theorem 10 in [14]).

The following corollaries are an immediate consequences of the above theorem.

Corollary 3.12. Let $\Lambda_{l}(l=1,2)$ be the linear functionals defined by (3.5) and (3.6) associated with family $\mathbb{F}_{2}$. Then the following statements hold:

(i) The function $v \mapsto \Lambda_{l}\left(f_{v}\right)$ is an exponentially convex function in the Jensen sense on J.

(ii) If the function $v \mapsto \Lambda_{l}\left(f_{v}\right)$ is continuous on $\mathrm{J}$, then it is exponentially convex function on $\mathrm{J}$.

Corollary 3.13. Let $\Lambda_{l}(l=1,2)$ be the linear functionals defined by (3.5) and (3.6) associated with family $\mathbb{F}_{3}$. Then the following statements hold:

(i) If the function $v \mapsto \Lambda_{l}\left(f_{v}\right)$ is continuous on $\mathrm{J}$, then it is 2-exponentially convex function on $\mathrm{J}$. If $v \mapsto \Lambda_{l}\left(f_{v}\right)$ is additionally strictly positive, then it is log-convex on J. Furthermore, for every choice $\mathrm{q}, \mathrm{u}, \mathrm{w} \in \mathrm{J}$, such that $\mathrm{q}<\mathrm{u}<w$, Lypunov's inequality holds:

$$
\left[\Lambda_{l}\left(f_{\mathfrak{u}}\right)\right]^{w-q} \leqslant\left[\Lambda_{l}\left(f_{q}\right)\right]^{w-u}\left[\Lambda_{l}\left(f_{w}\right)\right]^{\mathfrak{u}-q} .
$$

(ii) If the function $v \mapsto \Lambda_{l}\left(f_{v}\right)$ is strictly positive and differentiable on $\mathrm{J}$, then for every $\mathrm{p}, \mathrm{q}, \mathrm{u}, w \in \mathrm{J}$, such that $\mathrm{p} \leqslant \mathrm{u}$ and $\mathrm{q} \leqslant w$, we have

$$
\mu_{\mathfrak{p}, \mathfrak{q}}\left(\Lambda_{l}, \Phi\right) \leqslant \mu_{\mathfrak{u}, w}\left(\Lambda_{l}, \Phi\right)
$$

where

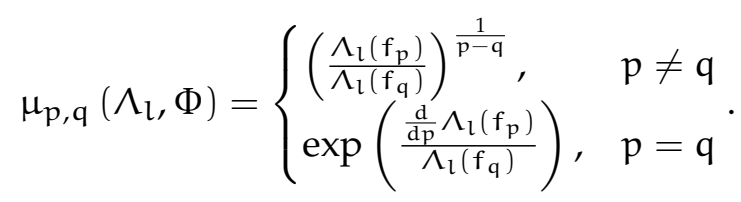

Proof.

(i) This is an immediate consequence of Theorem 3.11 and Remark 3.8.

(ii) Fix $l=1,2$, since $v \mapsto \Lambda_{l}\left(f_{v}\right)$ is positive and continuous, by (i) we have that the function $v \mapsto \Lambda_{l}\left(f_{v}\right)$ is $\log$-convex on J. So, for $p, q, u, w \in J$, such that $p \neq q$ and $u \neq w$ and $p \leqslant u$ and $q \leqslant w$, we have

$$
\frac{\log \Lambda_{l}\left(f_{p}\right)-\log \Lambda_{l}\left(f_{q}\right)}{p-q} \leqslant \frac{\log \Lambda_{l}\left(f_{u}\right)-\log \Lambda_{l}\left(f_{w}\right)}{u-w},
$$

i.e., we conclude that

$$
\mu_{\mathrm{p}, \mathrm{q}}\left(\Lambda_{\mathrm{l}}, \Phi\right) \leqslant \mu_{\mathrm{u}, w}\left(\Lambda_{\mathrm{l}}, \Phi\right) .
$$

Cases $p=q$ and $u=w$ follows from (3.12) as limiting cases. 
Remark 3.14. Note that the results from Theorem 3.11, Corollary 3.12 and Corollary 3.13 still hold when two of the points $z_{0}, \ldots, z_{\mathrm{t}} \in[\alpha, \beta]$ coincide, say $z_{1}=z_{0}$, for a family of differentiable functions $f_{v}$ such that the function $v \mapsto\left[z_{0}, \ldots, z_{t}, \frac{f_{v}(x)}{x}\right]$ is an n-exponentially convex in the Jensen sense (exponentially convex in the Jensen sense, log-convex in the Jensen sense), and furthermore, they still hold when all $(t+1)$ points coincide for a family of $t$ differentiable functions with the same property. The proofs are obtained by (1.15) and suitable characterization of convexity.

Remark 3.15. We can give the similar results as Theorem 3.11, Corollary 3.12, Corollary 3.13 and Remark 3.14 for $(2 t+1)$-points as to prove $(2 n)$-exponentially convex functions.

\section{Applications}

In this section, we give some applications of our generalized results about the upper bounds as well as exponential convex functions.

Firstly, we consider some related inequalities by using our generalized results of upper bounds.

Example 4.1. By using Ostrowski-type inequality (2.19) for $n=3$ as an upper bound of our generalized results,

- let $f(x)=e^{x}, x \in \mathbb{R}$, then

$$
0 \leqslant 1 \sum_{i=1}^{m} w_{i} e^{x_{i}}-\sum_{i=1}^{m} w_{i} e^{y_{i}} \mid \leqslant \frac{(\beta-\alpha)^{5}}{u^{\frac{1}{u}}}\left(e^{u \beta}-e^{u \alpha}\right)^{\frac{1}{u}}\left\|G_{p}\right\|_{v}
$$

- let $f(x)=x^{r},[0, \infty)$ for $r>1$, then

$$
\begin{aligned}
& 0 \leqslant\left|\sum_{i=1}^{m} w_{i} x_{i}^{r}-\sum_{i=1}^{m} w_{i} y_{i}^{r}\right| \\
& \leqslant(\beta-\alpha)^{5} \frac{r(r-1)(r-2)(r-3)(r-4)(r-5)}{(u(r-6)+1)^{\frac{1}{u}}}\left(\beta^{\mathfrak{u}(r-6)+1}-\alpha^{\mathfrak{u}(r-6)+1}\right)^{\frac{1}{u}}\left\|G_{p}\right\|_{v}
\end{aligned}
$$

- let $f(x)=x \log x, x \in(0, \infty)$, then

$$
0 \leqslant\left|\sum_{i=1}^{m} w_{i} x_{i} \log x_{i}-\sum_{i=1}^{m} w_{i} y_{i} \log y_{i}\right| \leqslant \frac{24(\beta-\alpha)^{5}}{(1-5 u)^{\frac{1}{u}}}\left(\beta^{1-5 u}-\alpha^{1-5 u}\right)^{\frac{1}{u}}\left\|G_{p}\right\|_{v},
$$

- let $f(x)=-\log x, x \in(0, \infty)$, then

$$
0 \leqslant\left|\sum_{i=1}^{m} w_{i} \log y_{i}-\sum_{i=1}^{m} w_{i} \log x_{i}\right| \leqslant \frac{120(\beta-\alpha)^{5}}{(1-6 u)^{\frac{1}{u}}}\left(\beta^{1-6 u}-\alpha^{1-6 u}\right)^{\frac{1}{u}}\left\|G_{p}\right\|_{v},
$$

where, $G_{p}=\int_{\alpha}^{\beta}\left(\sum_{i=1}^{m} w_{i} G_{p}\left(x_{i}, s\right)-\sum_{i=1}^{m} w_{i} G_{p}\left(y_{i}, s\right)\right) G_{3}^{\prime \prime}\left(\frac{s-\alpha}{\beta-\alpha}, \frac{t-\alpha}{\beta-\alpha}\right) d s,(p=1,2,3,4)$.

We can also give the particular cases of above results for $u=1$ and $v=\infty$. $(0, \infty)$.

Now, we construct exponentially convex function by using family of convex functions defined on

\section{Example 4.2. Let}

$$
\mathbb{E}_{1}=\left\{\theta_{v}:(0, \infty) \rightarrow(0, \infty): v \in \mathbb{R}\right\}
$$

be a family of continuous convex functions defined by 


$$
\theta_{v}(x)= \begin{cases}\frac{x e^{v x}}{v^{2}}, & v \neq 0 \\ \frac{x^{3}}{2}, & v=0\end{cases}
$$

We have $v \mapsto\left(\frac{\theta_{v}(x)}{x}\right)^{\prime \prime}(t \in \mathbb{R})$ is exponentially convex for every fixed $x \in \mathbb{R}$. Using analogous arguing as in the proof of Theorem 3.11 we also have that $v \mapsto \theta_{v}\left[z_{0}, \ldots, z_{t}\right]$ is exponentially convex (and so exponentially convex in the Jensen sense). Using Corollary 3.12 we conclude that $v \mapsto \Lambda_{l}\left(\theta_{v}\right)$ is exponentially convex in the Jensen sense. It is easy to verify that this mapping is continuous (although mapping $v \mapsto \psi_{v}$ is not continuous for $v=0$ ), so it is exponentially convex.

For this family of functions, $\mu_{v, q}\left(\Theta, \Lambda_{2}\right)$ from (3.11), becomes

$$
\begin{aligned}
& \mu_{\mathrm{t}, \mathrm{s}}\left(\mathbb{E}_{1}, \Lambda_{2}\right)=\left(\frac{\mathbb{E}_{1}\left(\theta_{\mathrm{t}}\right)}{\mathbb{E}_{1}\left(\theta_{s}\right)}\right)^{\frac{1}{\mathrm{t}-s}}, \mathrm{t} \neq \mathrm{s}, \mathrm{t}, \mathrm{s} \neq 0 ; \\
& \mu_{\mathrm{t}, \mathrm{t}}\left(\mathbb{E}_{1}, \Lambda_{2}\right)=\exp \left(\frac{\sum_{i=1}^{n} p_{i} x_{i}^{2} e^{\mathrm{t} x_{i}}-\sum_{i=1}^{n} p_{i} y_{i}^{2} e^{\mathrm{t} y_{i}}}{\sum_{i=1}^{n} p_{i} x_{i} e^{\mathrm{t} x_{i}}-\sum_{i=1}^{n} p_{i} y_{i} e^{t y_{i}}}-\frac{2}{t}\right), t=s \neq 0 ; \\
& \mu_{0,0}\left(\mathbb{E}_{1}, \Lambda_{2}\right)=\exp \left(\frac{1}{3} \frac{\sum_{i=1}^{n} p_{i} x_{i}^{4}-\sum_{i=1}^{n} p_{i} y_{i}^{4}}{\sum_{i=1}^{n} p_{i} x_{i}^{3}-\sum_{i=1}^{n} p_{i} x_{i}^{3}}\right) .
\end{aligned}
$$

Now using (3.10), $\mu_{t, s}$ is monotone function in parameters $t$ and $s$.

We observe here that $\left(\frac{\frac{d^{2} \theta_{t}}{d x^{2}}}{\frac{d^{2} \theta_{s}}{d x^{2}}}\right)^{\frac{1}{t-s}}(\ln x)=x$, so using Theorem 3.5 it follows that

$$
M_{t, s}\left(\mathbb{E}_{1}, \Lambda_{2}\right)=\ln \mu_{t, s}\left(\mathbb{E}_{1}, \Lambda_{2}\right)
$$

satisfies

$$
\alpha \leqslant M_{t, s}\left(\mathbb{E}_{1}, \Lambda_{2}\right) \leqslant \beta .
$$

This shows that $M_{t, s}\left(\mathbb{E}_{1}, \Lambda_{2}\right)$ is mean. Because of the above inequality (3.10), this mean is also monotonic. Remark 4.3. We can construct other examples for exponentially convex functions as Example 4.2 for the families of continuous convex functions:

$$
\mathbb{E}_{2}=\left\{\mu_{\mathrm{t}}:(0, \infty) \rightarrow \mathbb{R}: t \in \mathbb{R}\right\}
$$

where,

$$
\mu_{t}(x)= \begin{cases}\frac{x^{t+1}}{t(t-1)}, & t \neq 0,1 \\ -x \log x, & t=0 \\ x^{2} \log x, & t=1\end{cases}
$$

$$
\mathbb{E}_{3}=\left\{\chi_{\mathrm{t}}:(0, \infty) \rightarrow(0, \infty): \mathrm{t} \in(0, \infty)\right\}
$$

where,

$$
\begin{gathered}
\chi_{\mathrm{t}}(x)= \begin{cases}\frac{x \mathrm{t}^{-x}}{\log ^{2} \mathrm{t}}, & \mathrm{t} \neq 1 \\
\frac{x^{3}}{2}, & \mathrm{t}=1 .\end{cases} \\
\mathbb{E}_{4}=\left\{\delta_{\mathrm{t}}:(0, \infty) \rightarrow(0, \infty): \mathrm{t} \in(0, \infty)\right\}
\end{gathered}
$$

where,

$$
\delta_{t}(x):=\frac{x e^{-x \sqrt{t}}}{t}
$$




\section{Acknowledgment}

The research of the first author has been fully supported by HEC Pakistan. The research of the second author has been supported by the Royal Commission for Jubail and Yanbu, Kingdom of Saudi Arabia. This publication has been supported by the Ministry of Education and Science of the Russian Federation (the Agreement number No. 02.a03.21.0008.) The authors express their sincere thanks to the reviewers and editor for the useful suggestions to improve the paper.

\section{References}

[1] R. P. Agarwal, S. I. Bradanović, J. Pečarić, Generalizations of Sherman's inequality by Lidstone's interpolating polynomial, J. Inequal. Appl., 2016 (2016), 18 pages. 1, 2

[2] R. P. Agarwal, P. J. Y. Wong, Error Inequalities in Polynomial Interpolation and their Applications, Kluwer Academic Publishers, Dordrecht, (1993). 1, 1.8

[3] G. Aras-Gazić, V. Čuljak, J. Pečrić, A. Vukelić, Generalization of Jensen's inequality by Lidstone's polynomial and related results, Math. Inequal. Appl., 16 (2013), 1243-1267. 1, 2, 2

[4] P. Cerone, On Čebyšsv functional bounds, Differential \& difference equations and applications, 267-277, Hindawi Publ. Corp., New York, (2006). 1

[5] P. Cerone, S. S. Dragomir, Some new Ostrowski-type bounds for the Čebyšev functional and applications, J. Math. Inequal., 8 (2014), 159-170. 1

[6] K. R. Davidson, A. P. Donsig, Real Analysis with Real Applications, Prentice Hall, Upper Saddle River, (2002). 1

[7] L. Fuchs, A new proof of an inequality of Hardy-Littlewood-Polya, Mat. Tidsskr, B (1947), 53-54. 1

[8] G. H. Hardy, J. E. Littlewood, G. Pólya, Inequalities, Second ed., Cambridge University Press, London and New York, (1952). 1

[9] J. Jakšetić, J. Pečrić, Exponential convexity method, J. Convex Anal., 20 (2013), 181-197. 3

[10] S. Karlin, Total Positivity, Stanford Univ. Press, Stanford, (1968). 1

[11] A. R. Khan, N. Latif, J. Pečarić, Exponential convexity for majorization, J. Inequal. Appl., 2012 (2012), 13 pages. 3

[12] A. R. Khan, N. Latif, J. Pečarić, n-exponential convexity for Favard's and Berwald's inequalities and their applications, Adv. Inequal. Appl., 2014 (2014), 21 pages. 3

[13] M. A. Khan, N. Latif, I. Perić, J. Pečarić, On majorization for matrices, Math. Balkanica, 27 (2013), 3-19. 1.3

[14] K. A. Khan, J. Pečarić, I. Perić, Generalization of Popoviciu Type Inequalities for Symmetric Means generated by Convex Function, J. Math. Comput. Sci., 4 (2014), 1091-1113. 3, 3, 3, 3

[15] N. Mahmood, R. P. Agarwal, S. I. Butt, J. Pečarić, New Generalization of Popoviciu type inequalities via new Green functions and Montgomery identity, J. Inequal. Appl., 2017 (2017), 17 pages. 1, 1, 1

[16] A. W. Marshall, I. Olkin, B. C. Arnold, Inequalities: Theory of Majorization and Its Applications, Second Ed., Springer, New York, (2011). 1

[17] J. Pečarić, F. Proschan, Y. L. Tong, Convex functions, Partial Orderings and Statistical Applications, Academic Press, Boston, (1992). 1, 1, 1.8, 2, 2, 3

[18] T. Popoviciu, Sur l'approximation des fonctions convexes d'ordre superier, Mathematica, 10 (1934), 49-54. 1

[19] D. V. Widder, Completely convex function and Lidstone series, Trans. Amer. Math. Soc., 51 (1942), 387-398. 1 